\title{
Analysis of load of a powered roof support's hydraulic leg
}

\author{
Dawid Szurgacz ${ }^{1, *}$ and Jarosław Brodny ${ }^{2}$ \\ ${ }^{1}$ Polska Grupa Górnicza S.A., KWK ROW ruch Chwałowice, 4 Przewozowa St., 44-206 Rybnik, Poland \\ ${ }^{2}$ Silesian University of Technology, 26-28 Rossevelt St., 41-800 Zabrze, Poland
}

\begin{abstract}
The main purpose of the powered roof support is to protect headings from the impact of the rock mass. The result of such impact is static and dynamic load impacting the support section, which is carried by its construction. The basic elements of the construction of the support are hydraulic legs, whose task is to ensure adequate strength of its setting. Particularly in the case of dynamic impact of the rock mass, these legs are exposed to a very unfavourable load condition. Therefore, it is necessary to conduct tests to determine the parameters of operation for this type of loads. The paper presents the results of tests on the hydraulic leg subjected to impact load with free falling impact mass. The purpose of the research was to determine the parameters of the leg's operation, i.e. the time periods of pressure in the space under the piston and other elements of the hydraulic system. The tests were conducted in compliance with designed methodology and included innovative registration system. The obtained results clearly indicate the correctness of the adopted assumptions. According to the authors, the results should be applied during selection and operation of a powered roof support.
\end{abstract}

\section{Introduction}

The use of a powered roof support is largely dependent on the design features of the hydraulic leg the support is equipped with. The task of the leg is to take over the pressure of the floor rocks caused by the static clamping of the excavation, or the dynamic load caused by a mining tremor. The resulting dynamic load during the tremor, impacting on the powered roof support may in the first place cause damage to the hydraulic legs. From a physical point of view, the tremor can be treated as an earthquake caused by mining activities. The threat of mining tremors is one of the most common natural hazards the mining industry must face. The increase in the risk of rock mass tremors is directly influenced by the increased depth of exploitation and operational occurrences occurring in the overlying seams [1].

Deteriorating conditions in the majority of mines in the world [2-4] cause that the powered roof support is increasingly exposed to dynamic loads. The size and characteristics of these burdens are not sufficiently recognized at present which increases difficulty in predicting the effects of such burdens. This is mainly caused by complex research conditions. As a result, the loads are assessed mainly on the basis of their consequences, which in many cases include large damage to the legs. It should also be emphasized that the selection process of the legs for the support and the roof support for the longwall excavations should include the ventilation hazards and impact of the tremor, which has a significant impact on the risk of endogenous fires [5-8].
Currently, in order to protect the roof support's leg from damage caused by dynamic load, it is appropriately yielded. The process mainly refers to the selection of the appropriate safety valve. The purpose of such valve is to protect the leg from overloading, that means the pressure in the cylinder is larger than assumed. The valve operation starts when the pressure in the leg exceeds the permissible value, i.e. the values that are safe for the leg. This value results from the design and strength properties of the leg. After exceeding certain parameters, the safety valve opens and the fluid flows out of the leg. The fluid stops flowing through the valve when the pressure of the medium reaches the pressure necessary to balance the forces acting in the valve. In the case of the short dynamic loads, the valve parameters should allow the valve to be opened quickly in order to protect the leg against overload. For this reason, proper selection of the valve, and later the leg for the powered roof support section, is a complicated task requiring appropriate knowledge and experience. In order to obtain this knowledge, tests of hydraulic legs, with various safety valves, are carried out to determine the impact of the load on the characteristics of their operation.

The paper focuses on determining the operating parameters of the hydraulic leg under dynamic load. The source of the load on two tests stations was the impact of free falling mass, a drop-weight test, which reflected the dynamic load of the rock mass. The tests were also carried out at a station where the source of the load was an explosion of an explosive material. The paper presents the documentation developed during the tests. An innovative measuring and recording system was used

\footnotetext{
* Corresponding author: dawidszurgacz@vp.pl
} 
to register data $[9,10]$. Properly prepared hydraulic legs (Hydrotech) were subjected to tests.

\section{Dynamic Research Test Bench}

Dynamic tests of hydraulic legs are carried out in Poland by three research centres, the Central Mining Institute, TLO Opava and KOMAG. The first two centres offer tests based on drop-weight testers, while IMT KOMAG has a dynamic testing station using the explosive method. In the case of drop-weight tests, the load is a freely falling impact mass. Figure 1 presents the dropweight station at the Central Mining Institute in Katowice, Poland. First, the leg is placed (2) on the foundation of the station (1). The leg (2) rests on the traverse (3), which is locked with bolts (4). The remaining elements of the station are guides (5), on which the impact mass moves (6). A safety valve (7) and a pressure sensor (8) are connected to the under-space of the piston of the leg. The recording of the test results was carried out by an innovative measurement and recording system (9). The Opava TLO's leg construction is similar as presented in Figure 2.

In the case of a system for measuring and recording the work parameters of the leg, the new innovative construction was used for measurements. This system comprises a control and intermediary module that allows testing of the operation of individual control elements of the powered roof support. The system was developed using the National Instruments cRIO9030 card. This module is equipped with two different operator interface devices. Communication with the system is based on an Ethernet link. A PC computer that is equipped with software made in the Lab View environment can be used for this purpose. During the test, the leg with the safety valve slides down under the influence of an external load. The purpose of the valve is to prevent exceeding the pressure in the under-piston space of the leg. This in turn is supposed to prevent the growth of forces that can damage or destroy the leg.

Measurement of the liquid pressure in the leg without the safety valve and in the leg with the safety valve loaded with the same impact energy shows the efficiency of the valve operation. In the case of a system without a safety valve, the leg may be damaged. For this reason, in the presented studies, no such measurement was made. The kinetic energy of the impact (or potential falling mass) transmitted by the drop weight to the tested leg partially changes the leg slid, which results from the elastic properties of the liquid and the opening of the tested safety valve. The remaining part changes into the energy of elastic deformation of the leg's cylinder, while the rest of the energy is dispersed due to the vibrations of the test leg. From the research and practical point of view, the most important is the maximum pressure in the space under the leg's piston. which impacts on the load value. The time course of the change in the value of this pressure is also very important. It can be assumed that this course determines the characteristics of the leg's work and its entire hydraulic system. The character of the course is determined by the parameters of the safety valve's operation, i.e. its efficiency, as well as the opening and closing time.

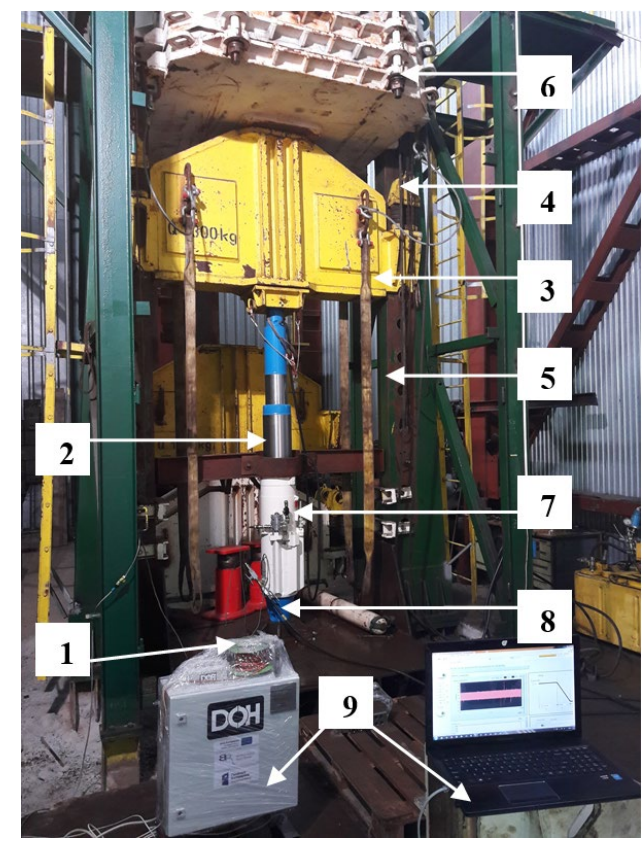

Fig. 1. A drop-weight tester at the Central Mining Institute: 1) testing station, 2) hydraulic leg, 3) traverse, 4) bolts, 5) guides, 6) impact mass, 7) safety valve, 8) pressure sensor, 9) recording system.

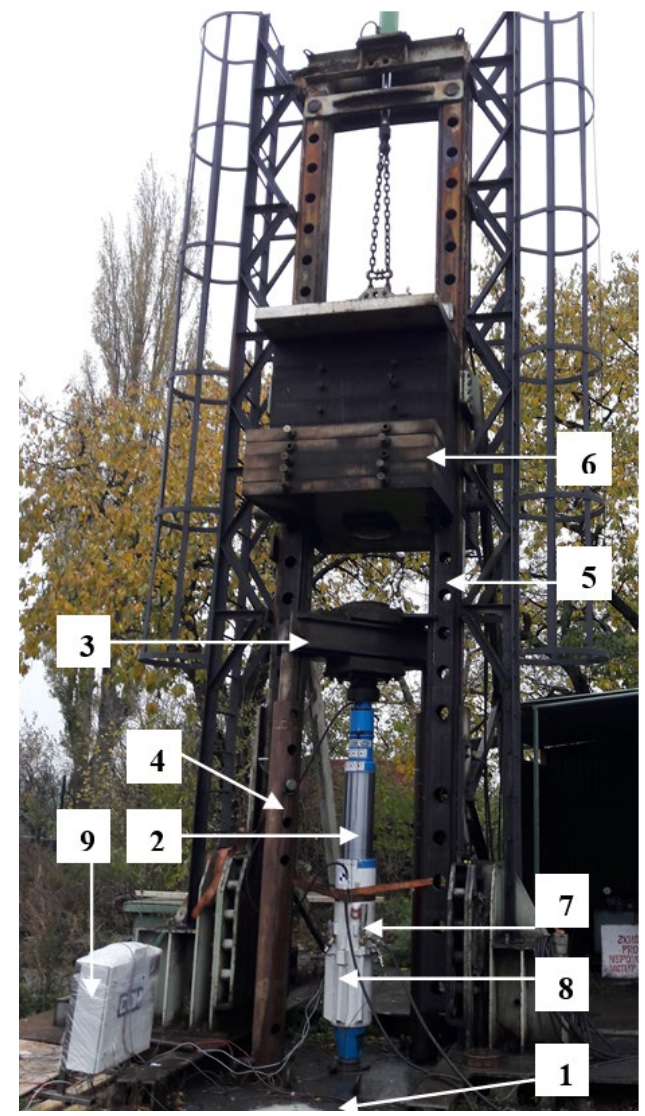

Fig. 2. Dynamic research tester in TLO Opava: 1) testing station, 2) hydraulic leg, 3) traverse, 4) bolts, 5) guides, 6) impact mass, 7) safety valve, 8) pressure sensor, 9) recording system. 
The testing station located at the KOMAG Institute of Mining Technology is also worth mentioning when describing locations where leg tests under dynamic loads are carried out. The station is shown in Figure 3.

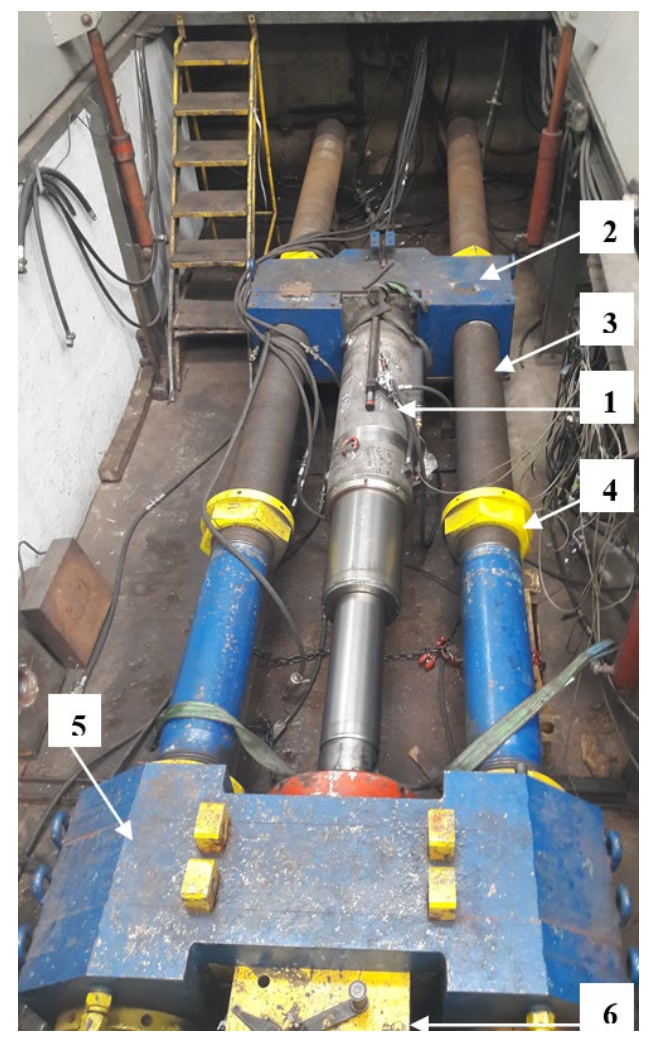

Fig. 3. Dynamic research station at ITG KOMAG: 1) hydraulic leg, 2) traverse, 3) bolts, 4) lockable nuts, 5) fixed nuts.

The station uses an explosion as the source of the load. The tested hydraulic leg (1) is slid between the traverse - mobile (2), connected by bolts (3) and lockable nuts (4) and fixed (5). The fixed traverse has a built-in dynamic load generator (6). The basic element of the generator is a piston placed in the cylinder with a built-in loading chamber, in which the powder charges are placed in the shells. The closing of the loading compartment is carried out using an artillery lock. The gas pressure resulting from the combustion of the explosive acts on the piston, which loads the test leg. The maximum value of the force with which the piston acts on the tested object is regulated by changing the density of the generator's chamber. The times of rise and duration of the load depend on the type and granulation of the explosive.

Also, in this case, the tests are aimed at determining the performance characteristics of the tested leg. However, the distribution of forces and the dissipation of dynamic energy are different to some extent. The work presents the test results of the leg together with the safety valve carried out at the drop-weight station at TLO Opava.

\section{Results and discussions}

The subject of dynamic research was a hydraulic leg of a powered roof support equipped with a safety valve. The research focused mainly on the effectiveness of the safety valve at the moment of dynamic load impacting on the leg. The efficiency of the valve operation at the moment of loading the leg is defined as the ratio of the maximum pressure of the liquid in the space under the piston of the leg against the energy of the impact mass. The kinetic energy of the impact is determined on the basis of the weight of the drop weight and the height from which this mass falls. This in turn determines the velocity at which the impact mass touches the leg. It can be assumed that with constant parameters of the entire system, the speed of displacement of the piston of the hydraulic leg caused by the energy of the impact increases with the increase of this energy. This state corresponds to the actual conditions. It is assumed that during a tremor in the longwall working, the speed of movement of the pistons of individual legs are equal to the speed of the rock mass. However, the movement of the piston of the hydraulic leg causes the fluid from the cylinder of the leg to be pushed out. The same results were obtained during tests. In this case, the fluid must flow out of the under-piston space through the safety valve. A number of such cases were observed in the course of carried out stationary tests.

Figure 4 presents three tested legs for which the outflow of fluid from the space under the piston leg was registered. These tests were carried out for the impact mass falling from a height, respectively: $0.3 \mathrm{~m} ; 0.4 \mathrm{~m}$; $0.5 \mathrm{~m}$.

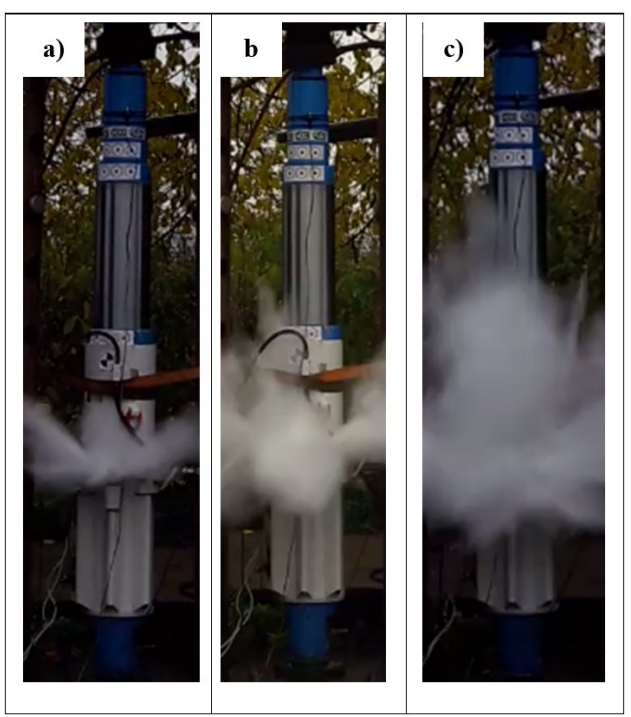

Fig. 4. Flow of fluid through the safety valve at the moment of impact from height: $\mathrm{a}=0.3 \mathrm{~m}, \mathrm{~b}=0.4 \mathrm{~m}, \mathrm{c}=0.5 \mathrm{~m}$.

As a result of this outflow, the piston of the actuator was dislocated and the pressure in the space under the piston of the tested actuator decreased. The setting of the safety valve was also very important during these tests. The setting is defined as the value of the pressure described as safe in the space under the piston of the 
actuator. Practice shows that the higher the value of this pressure, the more rigid the test system becomes. The research team assumed that the value of pressure setting the safety valve in the leg is set at $38 \mathrm{MPa}$ (380 bar). The value of the impact mass falling on the tested leg reached $2,000 \mathrm{~kg}$. The value of this mass also determined the values of the energy of the impact for which the tests were conducted. In the presented cases, these values reached respectively: $58.86 \mathrm{~kJ} ; 78.48 \mathrm{~kJ} ; 98.10 \mathrm{~kJ}$.

The main goal of the conducted research was to determine the dynamic performance of the dynamically loaded leg. This is referred to as the time course of the pressure build-up of the fluid in the under-piston space of the dynamically loaded leg.

The courses of changes in the value of this pressure recorded during the tests for individual cases are presented in Figures 5,6 and 7. The obtained waveforms show that several drops of the same impact mass were recorded. In the first two cases, there are clearly visible two maximum and slightly smaller thirds. In the third case, three local maxims of pressure values in the space under the piston leg are clearly visible. This is the result of the elasticity of the entire testing system. It seems that in real conditions such phenomena also occur. This is caused by vibrations of the rock mass and its interaction with the hydraulic system of the support.

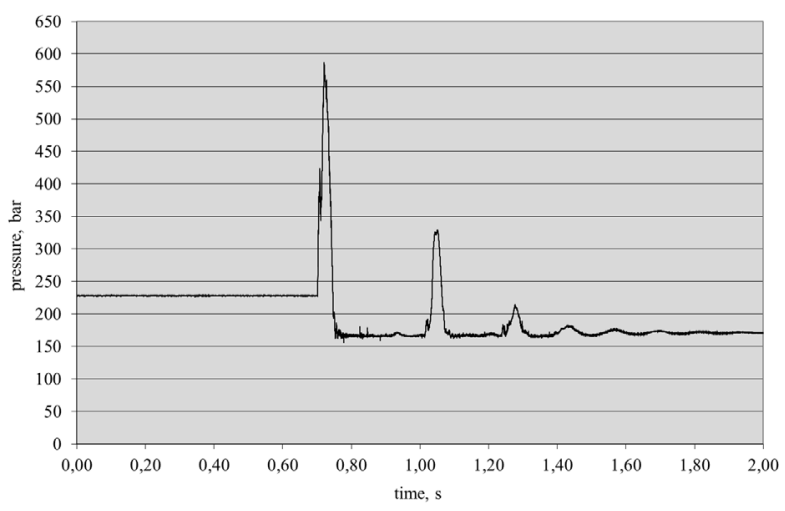

Fig. 5. Time course of the maximum value of the fluid pressure in the under-piston space of the leg during impact tests $(\mathrm{h}=0.3$ $\mathrm{m})$.

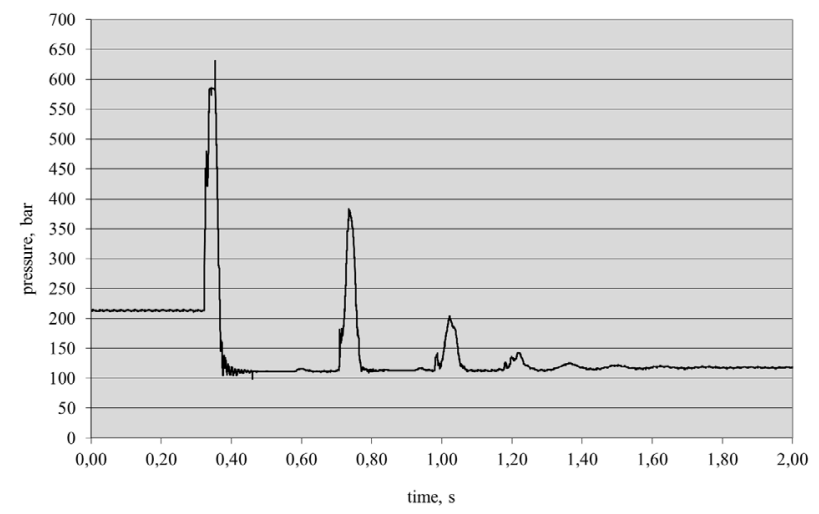

Fig. 6. Time course of the maximum value of the fluid pressure in the under-piston space of the leg during impact tests $(\mathrm{h}=0.4$ $\mathrm{m})$.

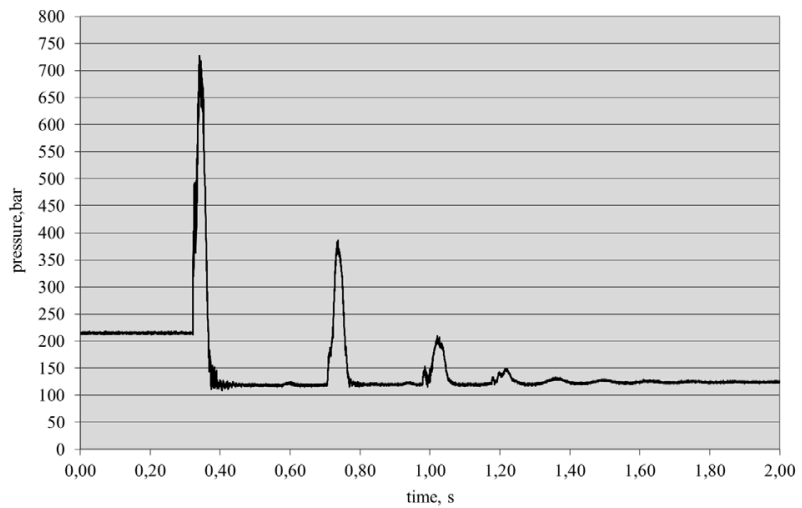

Fig. 7. Time course of the maximum value of the fluid pressure in the under-piston space of the leg during impact tests $(\mathrm{h}=0.5$ $\mathrm{m})$.

On the basis of the conducted tests, the value of the safety valve effectiveness index for these tests was also determined. As already mentioned, it defines the ratio of the maximum pressure of the fluid in the space under the piston of the leg to the energy of the impact. The results are presented in Figure 8.

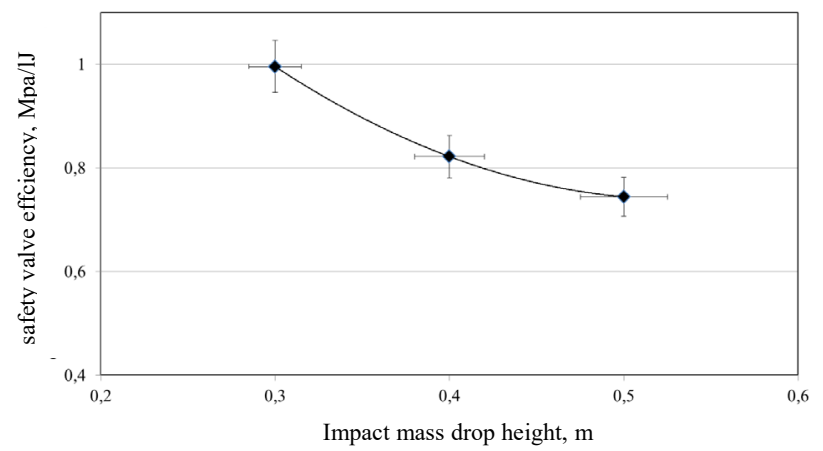

Fig. 7. Time course of the maximum value of the fluid pressure in the under-piston space of the leg during impact tests $(\mathrm{h}=0.5$ $\mathrm{m})$.

\section{Conclusions}

The authors of the research are aware that there is a difference between natural conditions and conditions occurring during tests conducted in a testing station. However, these tests were undertaken to determine the time course of the pressure change in the leg and to determine the effectiveness of the safety valve. At the same time, the authors are aware of the fact that in real conditions there may be some differences in registered values. It is justified to conduct this type of research in order to learn the mechanism of dynamic phenomena and to deepen the knowledge about the reaction of the hydraulic leg in the case of such phenomena. In underground conditions, the weight of the rock material dynamically loading the roof support is usually greater than its load-bearing capacity, while the weight of the drop weight is smaller than the nominal load capacity of the tested leg. Also, the elasticity of the drop weight and the foundation of the station differ from the elasticity of 
the floor and roof rocks. There are also different values of velocity of rocks mass impacting on the roof support and legs in the excavation at the moment of a tremor. It seems, however, that the carried out research presented the essence of these complicated phenomena occurring in the rock mass. The results obtained are very valuable. Particularly due to the registered characteristics and the outflow of fluid through the safety valve.

The role of the innovative measurement and recording system in these studies should also be emphasized. This system can also be successfully used to monitor the roof support's operation process, which in turn can be used to test the effectiveness of the entire longwall system [11-13]. The obtained results also confirmed the huge role that the safety valve plays in the hydraulic system of the roof support. Despite the increase in the maximum pressure in the leg, it did not exceed $100 \mathrm{MPa}$, which should be considered a very good result. It can therefore be assumed that the tested valve fulfilled its task.

The developed methodology and presented research are the beginning of more advanced research that the authors plan to carry out. The goal will be to compare the efficiency of different types of safety valves at different load configurations.

According to the authors, carrying out tests for devices or machines operating underground should contribute to a better understanding and improvement of methods assessing the usefulness of laboratory test results for real conditions. The obtained test results will also be used for the analysis and construction of new structures of a powered roof support adapted to work in dynamic load conditions.

The work was carried out within the project 'Innovative electro-hydraulic control system for powered roof support' No. POIR.01.01.01-00-1129/15. The Operational Programme Smart Growth 2014 - 2020 carried out by the National Centre for Research and Development.

\section{References}

1. S. Prusek, S. Rajwa, A. Wrana, A. Krzemień, Int. J. Min. Rec. E., 31:8 (2017)

2. M. Blaschuk, A. Dronov, A. Koperchuk, R. Chernukhin, V. Litvienko, E3S Web Conf., 15 (2017)

3. G. Buyalich, K. Buyalich, M Byakov, E3S Web Conf., 21 (2017)

4. S. V. Klishin, V.I. Klishin, G. Yu. Opruk, J. Min. S., 49:6, 932 (2013)

5. M. Tutak and J. Brodny. IOP Conf. E. Env. S., 95 (2017)

6. M. Tutak and J. Brodny. IOP Conf. E. Env. S., 95 (2017)

7. M. Tutak. SGEM2017 Vienna GREEN Conf., 17, 43, 301-308 (2017)

8. M. Tutak and J. Brodny, E3S Web Conf., 29 (2018)

9. D. Szurgacz, E3S Web Conf., 29 (2018)
10. D. Szurgacz and J. Brodny, E3S Web of Conf., 29 (2018)

11. J. Brodny, S. Alszer, J. Krystek, M. Tutak. Arch. Con. S. 27(LXIII), 2, 197-209 (2017)

12. K. Stecuła, J. Brodny, M. Tutak. CBU Int. Conf. 5, 1215-1219 (2017)

13. J. Brodny and M. Tutak. E3S Web Conf., 29 (2018) 